\title{
Comunicación gubernamental y emociones en la crisis del Covid-19 en España
}

\section{Governmental communication and emotions in the Covid-19 crisis in Spain}

José Carlos Losada Díaz Universidad de Murcia. España.

jclosada@um.es

[CV] $C R^{\circ} D 0$

Leticia Rodríguez Fernández. Universidad de Nebrija. España.

lrodrigu@nebrija.es

$[\mathrm{CV}]$ (1) $\mathrm{R}^{\mathrm{C}} \mathrm{O}$

Francisco Javier Paniagua Rojano. Universidad de Málaga. España.

fjpaniagua@uma.es.

$[\mathrm{CV}] \mathrm{G} \mathrm{R}^{\mathrm{C}} \mathrm{O}$

Cómo citar este artículo / Referencia normalizada

Losada Díaz, J. C., Rodríguez Fernández, L. y Paniagua Rojano, F. J. (2020). Comunicación gubernamental y emociones en la crisis del Covid-19 en España. Revista Latina de Comunicación Social, 78, 23-40. https://www.doi.org/10.4185/RLCS-2020-1467

\section{RESUMEN}

Introducción. Se analiza el impacto de la información sobre el COVID-19 en la población española para identificar los efectos y las emociones relacionadas con los canales y fuentes de consumo informativo durante la primera fase de la pandemia, que coincidió con los primeros días del estado de alarma. Metodología. Se realizó una encuesta administrada a través de Internet $(n=1823$.) que se distribuyó durante la segunda y la tercera semana de confinamiento (del 23 de marzo al 8 de abril de 2020) a toda la población mayor de edad residente en España y con acceso a internet. Resultados. Entre los resultados obtenidos destacan que, las noticias sobre la pandemia generaban en ese periodo diferentes emociones negativas -Tristeza, Ansiedad, Miedo, Confianza e Ira-, que variaban en función del canal de consumo informativo. Entre los temas que más interés tuvieron para los encuestados destacan los datos sobre la evolución de la pandemia, las medidas de protección y las formas de contagio; mientras que las fuentes más creíbles fueron las organizaciones y las autoridades oficiales, el personal sanitario y los medios de comunicación. Se aprueba la gestión de comunicación de crisis realizada por el Gobierno, que obtiene una nota media de 7,5 durante esta primera fase de la crisis. Se demuestra una circulación amplia de noticias falsas y de desinformación. Conclusión. Se demuestra la importancia de las emociones -en especial, las negativas- en las percepciones de la población en contextos de crisis como la del Covid-19 en España. En concreto, el Apoyo Emocional es el segundo mensaje oficial mejor valorado y, la Empatía, la característica más destacada de la información recibida. Se aprecia positivamente la capacidad del Gobierno por "entender" y "ponerse en lugar de la ciudadanía" por delante de cualquier otro aspecto. 
PALABRAS CLAVE: comunicación; emociones; crisis; covid-19; medios de comunicación; desinformación; fake news; comunicación de crisis; comunicación corporativa.

\begin{abstract}
Introduction. The impact of the information on COVID-19 on the Spanish population is analysed to identify the effects and emotions related to the channels and sources of information consumption during the first phase of the pandemic, which coincided with the first days of the state of alarm. Methodology. To this end, a survey administered via the Internet $(n=1823$.) was distributed during the second and third weeks of confinement (from March 23 to April 8, 2020) to the entire elderly population resident in Spain and with access to the Internet. Results. Among the results obtained, it is worth noting that news about the pandemic generated different negative emotions during this period - sadness, anxiety, fear, confidence and anger - which varied depending on the channel of information consumption. Among the topics that were of most interest to those surveyed were data on the evolution of the pandemic, protective measures and forms of infection, while the most credible sources were organizations and official authorities, health personnel and the media. Conclusions. It is shown the importance of emotions - especially negative ones - in the population's perceptions in crisis contexts such as the Covid-19 in Spain. Specifically, Emotional Support is the second most valued and official message, empathy, the most outstanding feature of the information received. The Government's ability to "understand" and "put itself in the place of the citizenry" ahead of any other aspect is positively appreciated.
\end{abstract}

KEYWORDS: communication; emotions; crisis; covid-19; media; disinformation; fake news; crisis communication; corporate communication.

\title{
CONTENIDO
}

1. Introducción. Emociones, comunicación y públicos. 1.1. Emociones en la gestión de la comunicación de crisis de las organizaciones. 2. Objetivos y metodología. 3. Resultados. 3.1. Perfil y consumo informativo de los encuestados. 3.2. Emociones relacionadas con la pandemia y los medios de información. 3.3. Valoración de la comunicación de crisis realizada por el Gobierno. 4. Discusión y conclusiones. 5. Bibliografía.

\section{Introducción. Emociones, comunicación y públicos}

Hay un fuerte componente subjetivo en el modo en el que la gente interpreta las situaciones de crisis, la forma en la que éstas les pueden afectar y cómo protegerse ante ellas. Este conjunto de percepciones que manejan los públicos es decisivo para la gestión de una crisis y deben ser consideradas en todo momento en la toma de decisiones. De hecho, las investigaciones de los últimos años recuerdan que las crisis son lo que los públicos piensan sobre ellas y no tanto los hechos en sí. Así, Coombs (2007) afirma que la crisis “(...) puede verse como la percepción de un hecho que amenaza a los públicos y hasta el propio desempeño de la organización. En la misma línea, las crisis son en gran medida, perceptivas. Si los públicos creen que hay una crisis, la organización tiene una crisis, a no ser que les convenzamos de lo contrario (...)". De modo similar, Kleinnijenhuis et al.(2015) asegura: "(...) Incluso, se puede afirmar que, aunque las crisis tienen un origen real, al final se construyen a partir de la interacción entre varios actores, cuyas percepciones producen consecuencias reales". Y Utz et al. (2013): "En esta era de comunicación socialmente mediada, los públicos determinan la relevancia, el interés y la credibilidad de la información que reciben".

Este grupo de percepciones que dan forma a lo que los públicos opinan sobre las crisis poseen dos tipos de elementos: racionales y emocionales. De hecho, este segundo grupo es decisivo en la 
construcción mental de la población, como confirman Slovic y Burns (2012) para quienes, durante las crisis, "los receptores no sólo confían en lo que piensan acerca de un tema, sino también en lo que sienten sobre ese tema". De hecho, según asegura Dunlop et al. (2008) "los individuos necesitan asociar algún tipo de etiqueta emocional a un problema para sentir riesgo por ello". Según Pang, Jin y Cameron (2009), "en una crisis, a medida que el conflicto entre el público y la organización se intensifica, las emociones son uno de los anclajes que utilizan los públicos para interpretar lo que está pasando". La carga emocional en una crisis, como recuerdan Van der Meer et al. (2014), están presentes en un amplio conjunto de públicos, no solo en los directamente afectados: "(...) una crisis puede no solo ser una experiencia emocional para el público, sino también para el conjunto de la organización y de sus miembros".

La investigación de los últimos años demuestra el peso sustancial que las emociones tienen en los procesos mentales activados por los ciudadanos en momentos de crisis (Losada, 2018). Las emociones están presentes en la interpretación de los hechos que generan la crisis, pero, del mismo modo, condicionan la interpretación que los públicos hacen sobre la responsabilidad en esos hechos $\mathrm{y}$, por supuesto, la respuesta institucional que adopta la organización en cada momento. Este conjunto de interpretaciones de carácter emocional acaba teniendo un peso sustancial en la evolución de la crisis, debido a la conexión directa entre estas percepciones y las conductas finales que deciden adoptar los ciudadanos. Para Choi y Lin (2017), Kim y Cameron (2011) o Van der Meer et al. (2014), "la emoción comunicada puede afectar la interpretación del público y, como consecuencia, a la efectividad de la respuesta a la crisis".

La conexión entre las emociones de los públicos y sus reacciones se recogen en varios trabajos centrados en la atribución que los públicos realizan sobre la responsabilidad de la crisis, como los liderados por Coombs (2004), Coombs y Holladay, (1996), (2001) y (2004) y el modelo SCCT (Situational Crisis Communication Theory). En la medida en la que se demuestra la responsabilidad de una organización en una situación de crisis, se intensifican los sentimientos de ira y de angustia y disminuyen los de simpatía, lo que puede acabar con un ataque directo a la organización, tanto en el relato que hagan sobre ella (el «boca a boca» entre individuos que tanto peso ha ganado con las redes sociales) como en la ruptura de relaciones con la marca. $\mathrm{O}$, incluso, en la disposición a protagonizar conductas que van en contra de la voluntad de la organización o hasta contra la propia seguridad de los afectados. Pero, la atribución de responsabilidad en la crisis no es la única opción en la construcción de emociones y muchos otros académicos distinguen aún más entre las emociones independientes de la atribución y las dependientes de la atribución en el contexto de las crisis organizacionales (Choi y Lin, 2017).

Un buen número de investigaciones se centran en localizar las emociones más habituales en contextos de crisis. Una de las aportaciones más relevantes es la de Jin, Liu, Anagondahalli y Austin (2014), quienes propusieron una escala para medir las emociones de los públicos ante una situación de crisis. Los resultados fueron una lista de 13 emociones concretas, casi todas ellas negativas: ira, ansiedad, aprensión, confusión, desprecio, disgusto, vergüenza, miedo, culpa, tristeza, bochorno, sorpresa y simpatía. Estas emociones se pondrían en marcha de formas diferentes, dependiendo del modo en el que los públicos enfocan la responsabilidad de los hechos: algunas de estas emociones eran independientes a cualquier tipo de atribución (como la ansiedad o el miedo), otras dependían de la atribución externa de la responsabilidad, es decir, a una empresa (como la ira, el desprecio o la tristeza...) y otras, finalmente, se activaban principalmente a partir de una atribución interna de la responsabilidad - a sí mismos- (como los sentimientos de culpa, vergüenza o bochorno).

Previamente, Pang, Jin y Cameron (2010) reducían a cuatro las emociones principales que se ponen en marcha en los públicos primarios ante una situación de esta naturaleza. Estas emociones serían 
Ira, Miedo, Ansiedad y Tristeza. La Ira se activaría, según estos autores, porque la gente se siente amenazada ante una organización que va contra ellos y contra su bienestar, por lo que se culpa a la compañía responsable de las acciones dañinas, que podría haber controlado o impedido en su momento. Por esta razón, la gente aumenta este "enfado", que puede transformarse con facilidad en un comportamiento beligerante contra la organización; por otra parte, el Miedo se activaría ante la incertidumbre del público, que no está seguro de cómo reaccionar y cómo la organización va a manejar la situación (de hecho, muchas veces, dependiendo de sus recursos y de su poder, pueden elegir evitar o escapar de la crisis); en cuanto a la Ansiedad, esta emoción se derivaría, según afirma Lazarus (1991), de la presencia de un peligro inmediato y concreto. El público puede sentirse abrumado por la situación y buscar las soluciones inmediatas. De la misma forma, pueden culpar o no a la organización según otras variables; por último, la Tristeza, que emergería cuando el público sufre una pérdida tangible o intangible o ambas cosas (estima, valores morales, ideal, personas y su bienestar, etc.), de la que no puede culpar a nadie, por lo que necesitará cierto alivio o consuelo, aunque es cierto que, si la pérdida puede ser restaurada o compensada en alguna medida, la Tristeza puede llegar a asociarse a la esperanza. Esto dependerá, sobre todo, de las medidas que la organización vaya tomando. En todo caso, la mayor parte de los estudios se centran en emociones negativas, que parecen tener un rol más importante que las positivas en contextos de crisis. En esa línea están los trabajos centrados en la Ira ( $\underline{\mathrm{Utz}}$ et al., 2013 o Van der Meer et al., 2014), la Vergüenza y el Arrepentimiento (Wesseling et al. (2006) o el Miedo (McDonald et al., 2010).

En todos los casos, los diferentes autores destacan la importancia de contemplar las emociones surgidas en una crisis de forma estratégica y no como una mera amenaza. Pang, Jin y Cameron (2009 y 2010), aseguran que gestionar adecuadamente las emociones negativas puede lograr revertir la situación inicial e, incluso, mejorar el compromiso entre la organización y sus públicos. Éstos pueden sentir alguna de estas emociones o pueden pasar de unas a otras dependiendo de varios factores. La forma más visible en el que la organización puede gestionar las emociones negativas y contrarrestarlas será con su propia respuesta desde el primer momento, de su gestión y de su comunicación. Para ello, es imprescindible que sea especialmente sensible con la situación real, comunicando con "compasión, preocupación y empatía” (Heath, 2006).

\subsection{Emociones en la gestión de la comunicación de crisis de las organizaciones}

Álvarez (2009) destaca que la gestión moderna de las crisis pasa, sobre todo, por el estudio de los públicos, situando el foco de atención en sus percepciones y en el modo en el que reciben la información que les llega, la canalizan emocionalmente, la interpretan y, con arreglo a todo ello, se comportan. Es una opinión que también sustenta Schwarz (2019), para quien la calidad de las relaciones con los públicos entre una organización y sus stakeholders tiene un impacto sustancial en las evaluaciones y atribuciones de responsabilidad hacia una organización.

En una situación de crisis, los públicos tienen una elevada predisposición a sentirse "maltratados" si no se sienten atendidos, activando todos los resortes que hacen visible esa sensación. De ahí surge la necesidad de mostrar una especial sensibilidad con estos grupos, que son la base sobre la que construyen las percepciones más sólidas y generales sobre los hechos que ocurren y que, en buena medida, otorgarán una forma y una medida a la crisis. Para ello, la organización debe concentrarse en propuestas de acción concretas a partir de tres frentes: hacia la mente, empleando estrategias persuasivas hacia los públicos y aparcando la estrategia de "defensa" ante cualquier ataque; hacia el cuerpo, en la medida en la que pueden satisfacer las necesidades físicas de la gente durante las crisis; y, sobre todo, hacia el corazón de los públicos, tratando de comprender los trastornos emocionales de esas audiencias (conscientes del decisivo papel que las emociones desempeñan durante las crisis, al que ya nos hemos referido). Parece imprescindible que la organización actúe de forma transparente 
en los tres ejes y, de este modo, los públicos puedan percibir una actitud constructiva y positiva de la organización en la resolución de la crisis y en la protección de los ciudadanos, a partir de diversas posibilidades de actuación (Coombs y Holladay, 2008; Schultz et al., 2011).

A la vez, las compañías deben estar preparadas para poder recibir y procesar la información "sentida" -de carácter más sentimental- por parte de sus públicos. Es decir, escuchar y monitorizar sus percepciones a través de un amplio conjunto de herramientas, especialmente las virtuales, como reconoce (Macias et al., 2009): "el conjunto de posibilidades digitales favorecen especialmente el intercambio de información, de opiniones y la expresión emocional sobre las crisis", fórmulas que van más allá de las tácticas tradicionales de comunicación de las relaciones públicas, como comunicados de prensa o declaraciones públicas (Wang et al, 2010) y permiten un intercambio directo, inmediato y personal". "Durante las crisis, los públicos se vuelven hacia los medios sociales para encontrar en ellos una amplia variedad de información y apoyo, en especial, emocional" (Hilyard y et al. 2010; Stephens y Malone, 2005).

Otra fórmula complementaria por la que una organización puede gestionar adecuadamente las emociones sociales durante estos contextos de crisis es la de compartir experiencias, una oportunidad que se transforma en una forma de desahogo y alivio personal que los públicos necesitan y a la que la compañía no puede permanecer ajena. Cuando comparte, la gente se considera más próxima a los que sufren y en alguna medida, se sienten más cerca de la solución, hecho al que se refiere Wendling (2013): "Cuando las personas sufren el impacto de una crisis, pero al mismo tiempo se les asigna el papel activo para compartir información, pueden sentirse empoderadas e involucradas en la respuesta. Pasan de ser observadores pasivos o víctimas a convertirse en actores". Como señalan Stephens y Malone (2012): "En esencia, lo único que quiere la gente es compartir sus experiencias".

La organización debe participar en esta condición, la de compartir, abriendo sus medios a las diferentes opiniones propias y ajenas y esforzándose en eliminar cualquier barrera que impida que la gente pueda conversar, tanto a través de los recursos propios como de las herramientas "ganadas". Y para lograr este fin, los medios sociales se convierten en instrumentos poderosos que hacen más sencilla y operativa la forma en la que las compañías y públicos comparten información (Lachlan et al. 2016), como confirma Macias et al. (2009): “(..) las redes sociales pueden facilitar el intercambio de información, de opiniones y la expresión emocional sobre las crisis". Aunque, como aseguran Liu et al(2015) "no hay una única forma para el uso de medios sociales en la difusión de información durante una crisis, pero es necesario que exista una inversión continua para garantizar que se utilicen muchas formas diferentes de comunicación”.

\section{Objetivos y metodología}

El objetivo principal de este trabajo es analizar la influencia de la información oficial sobre el COVID-19 en la población española durante las primeras semanas del Estado de Alarma (marzoabril de 2020), tratando de identificar las emociones relacionadas con los canales y las fuentes de consumo informativo. Como objetivos específicos, se proponen, entre otros, los siguientes:

- Identificar los canales de comunicación más utilizados por la población para seguir la información sobre el COVID-19

- Localizar los temas que más interés suscitaron sobre el COVID-19

- Señalar las fuentes más creíbles para la población, así como el papel de la información oficial

- Conocer la valoración de la gestión de la comunicación del Gobierno en la crisis

- Analizar el comportamiento de la audiencia ante las noticias falsas y las acciones tomadas para contrastar la información 
Como técnica metodológica, se apostó por la encuesta, instrumento útil para describir las relaciones entre diferentes variables en una población -que se convierte en un ejemplo representativo del conjunto- (Boyle y Schmierbach, 2020), con el objetivo de indagar y extraer conclusiones sobre fenómenos generales -en este caso, la reacción de los españoles ante la información que recibían sobre la pandemia-, con el apoyo de procedimientos estadísticos que definen muestras representativas de universos más amplios, a su vez generalizables, y que ofrecen una foto fija fiable de un momento concreto (Eiroa y Barranquero, 2017). En concreto, se apostó por una encuesta administrada a través de Internet (Boyle y Schmierbach, 2020; Eiroa y Barranquero, 2017).

Se trata de un formato utilizado cada vez con más frecuencia, y cuyas ventajas principales son que no necesita la asistencia del encuestador y que se puede realizar, por tanto, con pocos recursos, en un corto periodo de tiempo y a gran escala si se cuenta con un grupo de investigadores que contribuya en su difusión, ya que puede compartirse con rapidez a través de las redes sociales y otros canales digitales. Este tipo de técnicas permite un filtro automático en el diseño de la muestra e incluso, al responderse en el anonimato y la privacidad, facilita la sinceridad del encuestado (Boyle y Schmierbach, 2020).

La encuesta, "técnica cuantitativa que intenta medir hechos opiniones, actitudes y comportamientos de la población, a partir del empleo de cuestionarios estandarizados en los que se recogen testimonios verbales o escritos de una muestra más o menos representativa de sujetos con respecto a una población más amplia" (Eiroa y Barranquero, 2017) requiere de trabajo importante en su diseño y definición, tanto en la muestra, como en el planteamiento de las preguntas y el momento en que se realizan.

En este trabajo exploratorio se estudian los efectos que la comunicación del Gobierno y la información difundida a través de los diferentes canales offline y online en la población española, durante las primeras semanas de confinamiento de la población, pocos días después del anuncio del Estado de Alarma por parte del Gobierno de España. El trabajo trata de analizar las principales variables del consumo de información por parte de la población y la percepción, especialmente emocional, que le proporcionaba la información recibida, considerando los diferentes canales utilizados en cada momento, la credibilidad de las fuentes a las que tenía acceso y la importancia de los aspectos de la información que recibía.

Se diseñó una encuesta online a través de la herramienta de Google Encuesta, estructurada en diez preguntas: Canales de comunicación utilizados por la población para informarse sobre la pandemia, Temáticas que más interesaban, Fuentes más creíbles en este momento, Valoración de la gestión de la comunicación por el Gobierno, así como Elementos que influyen en esa valoración de la información oficial, Importancia de los tipos de mensajes, Emociones que generaba la información oficial que se recibía y, finalmente, Comportamiento ante las noticias falsas. Estas cuestiones se completaron con los Datos Sociodemográficos de los encuestados -Edad, Formación y Comunidad autónoma de residencia en España-. 
Tabla 1.

\begin{tabular}{|c|c|}
\hline PREGUNTA & POSIBLES RESPUESTAS \\
\hline $\begin{array}{l}\text { ¿Cuáles son los canales de } \\
\text { comunicación qué más está utilizando } \\
\text { para informarse sobre la crisis del } \\
\text { Coronavirus/COVID-19)? (señale } 3 \\
\text { opciones máximo) }\end{array}$ & $\begin{array}{ll}- & \text { Facebook } \\
- & \text { Instagram } \\
- & \text { Twitter } \\
- & \text { Youtube } \\
- & \text { WhatsApp } \\
- & \text { Televisión } \\
- & \text { Radio } \\
- & \text { Prensa } \\
- & \text { Blogs } \\
- & \text { Páginas web oficiales } \\
- & \text { Teléfono de contacto } \\
- & \text { Otros }\end{array}$ \\
\hline $\begin{array}{l}\text { ¿Cuáles son los temas sobre esta Crisis } \\
\text { que más le interesan? (señale } 3 \text { opciones } \\
\text { máximo) }\end{array}$ & $\begin{array}{ll}\text { - } & \text { Forma de contagio } \\
\text { - } & \text { Protección propia } \\
\text { - } & \text { Síntomas de la enfermedad } \\
\text { - } & \text { Acciones prohibidas y permitidas en el decreto } \\
\text { - } & \text { Evolución de la pandemia: datos } \\
- & \text { Información social y entretenimiento } \\
- & \text { Seguimiento de la pandemia en otros países } \\
- & \text { Otros }\end{array}$ \\
\hline $\begin{array}{l}\text { ¿Cuáles son las fuentes de información } \\
\text { más creíbles para usted en este } \\
\text { momento? (señale } 3 \text { opciones máximo) }\end{array}$ & $\begin{array}{ll}- & \text { Familiares y amigos } \\
- & \text { Organizaciones oficiales y autoridades } \\
- & \text { Medios de comunicación y periodistas } \\
- & \text { Personal sanitario } \\
- & \text { Líderes de opinión } \\
- & \text { Expertos y científicos } \\
- & \text { OMS } \\
- & \text { Otros }\end{array}$ \\
\hline $\begin{array}{l}\text { ¿Cómo cree que está gestionando la } \\
\text { comunicación el Gobierno en la crisis } \\
\text { del COVID-19? (Valore de } 1 \text { a } 10, \\
\text { siendo } 10 \text { la máxima nota) }\end{array}$ & $1-10$ \\
\hline $\begin{array}{l}\text { Valore los siguientes aspectos sobre la } \\
\text { información oficial que le está llegando, } \\
\text { según esté: Muy de acuerdo, De } \\
\text { acuerdo, Poco de acuerdo, Nada de } \\
\text { Acuerdo }\end{array}$ & $\begin{array}{ll}\text { - } & \text { Claridad en la información } \\
\text { - } & \text { Veracidad } \\
\text { - } & \text { Transparencia } \\
\text { - } & \text { Rapidez en la información } \\
\text { - } & \text { Empatía por parte de autoridades y los medios }\end{array}$ \\
\hline $\begin{array}{l}\text { Valore la importancia que tiene para } \\
\text { usted este tipo de mensajes que está } \\
\text { recibiendo (Valore de } 1 \text { a } 7 \text {, siendo } 1 \\
\text { menos importante y } 7 \text { más importante) }\end{array}$ & $\begin{array}{l}\text { - } \quad \text { La información sobre lo que está ocurriendo } \\
\text { - } \quad \text { Soluciones sobre lo que está ocurriendo } \\
\text { - } \quad \text { Apoyo emocional }\end{array}$ \\
\hline
\end{tabular}


¿Qué emoción/es le genera la información que recibe de fuentes oficiales sobre el coronavirus?
- Tristeza

- Miedo

- Confianza

- Ira

- Tranquilidad

- Orgullo

- Desconfianza

- Alegría

- Incertidumbre
¿Es consciente de si le ha llegado algún bulo, noticias falsas o fakenews durante la presente crisis?
- Sí

$-\quad$ No

- No sabe/No contesta

- Familia y amigos

- Organizaciones oficiales y autoridades

¿A través de qué medios contrasta la información? (Señale 3 opciones máximo)

- Medios de comunicación y periodistas

- Personal sanitario

- Líderes de opinión

- No contrasto información

- No contesta

- Teléfono

- Redes sociales

En caso de dudas, ¿ha enviado consultas o preguntas a través de los canales oficiales de comunicación que existen? (Señale 3 opciones máximo)

- Correo electrónico

- Whatsapp

- Telegram

- APPs

- No he solicitado información

- Otros

Fuente: Elaboración Propia

Se optó por un procedimiento no probabilístico -personas con acceso a Internet-, establecido a juicio de los investigadores, utilizando el muestreo de "bola de nieve", lo que significa que se compartió el cuestionario en línea a través de las redes sociales (Twitter, Facebook, Linkedin e Instagram a partir de los perfiles de los autores, que suman una comunidad de 18.334 seguidores), del correo electrónico y del sistema de mensajería electrónica WhatsApp (de acceso prácticamente universal en España), explicando la motivación del cuestionario y solicitando a los encuestados que pasaran a sus contactos, familiares y amigos. (Baxter; Babbie, 2004). Previamente, se testó la encuesta realizando un envío a 15 personas para comprobar que la redacción de las preguntas era comprensible y no generaba confusión.

Algunos expertos han mostrado su preocupación por las bajas tasas de respuesta en este tipo de sondeos en el ámbito de la Comunicación y las Relaciones Públicas (Kent, Taylor y Turcilo, 2006; Van Ruler y Lange de, 2003; Karadjov, Kim y Karavasilev, 2000; Wu y Taylor, 2003), por lo que algunos autores, como Wu y Taylor (2003: 475) sugieren acciones adicionales para completar los datos. Esta cuestión fue resuelta en el presente estudio con la utilización del sistema de mensajería electrónica WhatsApp, como hemos mencionado, lo que facilitó un incremento de respuestas, que pueden considerarse en los márgenes aceptables para la obtención de resultados significativos para estudios similares (Karadjov et al., 2000; Kent et al., 2006; Venter, 2010; Wu y Taylor, 2003). 
El periodo de respuesta se acotó entre los días 23 de marzo y 8 de abril de 2020. En total se registraron 1.952 respuestas que fueron sometidas a un filtrado, de acuerdo con Morrow and Skolits (2014), para eliminar las emitidas por residentes fuera de España y por menores de edad. La muestra se redujo, por tanto, a 1.823 personas, de entre 18 y 82 años, con residencia en territorio español y acceso a internet.

Posteriormente, los datos se procesaron en un archivo Excel, para contabilizar las frecuencias de respuestas y, además, se realizó un cruce entre la pregunta sobre la emoción generada por la información oficial en los públicos con la cuestión sobre el uso preferente de fuentes de información, con el objetivo de identificar las diferencias en los efectos de la información que llega en función del canal de recepción y confirmar, así, la confianza de estos medios.

\section{Resultados}

\subsection{Perfil y consumo informativo de los encuestados}

El perfil medio de encuestado es una persona de entre 35 y 54 años (45\%) con estudios universitarios $(74,6 \%$.) Sus canales prioritarios de información sobre la crisis del Covid-19 son la televisión $(72,6 \%)$, la prensa $(48,9 \%)$ y las páginas web oficiales $(31,2 \%)$. Le siguen Twitter $(30,3 \%)$, la radio $(29,4 \%)$, WhatsApp $(26,3 \%)$ y Facebook $(21,3 \%)$. Otras redes sociales como Instagram $(5,87)$ o Youtube $(4,2 \%)$ recogen menor impacto, así como los blogs $(1,4 \%)$ o los teléfonos de contacto $(1,7 \%)$, que suponen una muestra poco significativa. No obstante, las redes sociales siguen ganando peso como canales de información en situaciones de crisis. Así, el 88,24\% de los encuestados indicaron entre sus respuestas alguna red social para informarse sobre la pandemia.

Se observa que la información institucional es altamente valorada durante la fase de crisis analizada: las páginas web oficiales figuran como el tercer canal de información más utilizado, pero, además, un $70 \%$ identifican las organizaciones oficiales y las autoridades como las fuentes de información más creíbles. Se añaden el personal sanitario (68,3\%), los medios de comunicación $(52,8 \%)$, familiares y amigos $(8,3 \%)$ y líderes de opinión $(6,8 \%)$.

En cuanto a los intereses informativos, la evaluación de la pandemia y sus datos $(76,6 \%)$ se registra como la primera preocupación, así como la protección frente al virus y la de su entorno (62,4\%), las formas de contagio $(41,7 \%)$ y la sintomatología de la enfermedad $(41 \%)$. En un segundo plano, quedarían las acciones prohibidas y permitidas por el Real Decreto 463/2020 Decreto $(39,6 \%)$ que regulaba el Estado de Alarma, el seguimiento de la pandemia en otros países $(27,9 \%)$ y la información social y de entretenimiento relacionada $(9,7 \%)$. Se incluyó además la posibilidad de respuesta libre, recogiéndose un 1,4\% de encuestados que de manera espontánea incluyeron la economía entre sus principales preocupaciones.

Dado que la desinformación ha sido uno de los temas más controvertidos durante esta crisis y uno de los retos que deben ser abordados por los profesionales de la comunicación (Rodríguez-Fernández, 2019), se consideró pertinente añadir esta cuestión, identificando además los mecanismos de verificación utilizados. Un $93 \%$ de los encuestados asegura haber recibido fake news o bulos durante esta fase de la pandemia, porcentaje ciertamente muy elevado. Al consultar por las fuentes utilizadas para contrastar la información, el $70 \%$ señaló las organizaciones oficiales y autoridades en primer lugar, los medios de comunicación (65\%), el personal sanitario (50\%) y con mayor distancia líderes de opinión $(7,85 \%)$ y familiares y amigos $(5,60 \%)$. Solo un 3,18\% de la muestra reconocía no contrastar información. Este porcentaje es muy reducido, sobre todo al compararlo con los mecanismos de consulta en caso de dudas, pregunta en la que el 68,94\% de los encuestados 
reconocía no realizar consultas a canales oficiales, mientras que aquellos que sí que la realizan tienden a utilizar WhatsApp (13,28\%), el teléfono $(12,29 \%)$, las redes sociales $(10,65 \%)$ y el correo electrónico $(6,15 \%)$.

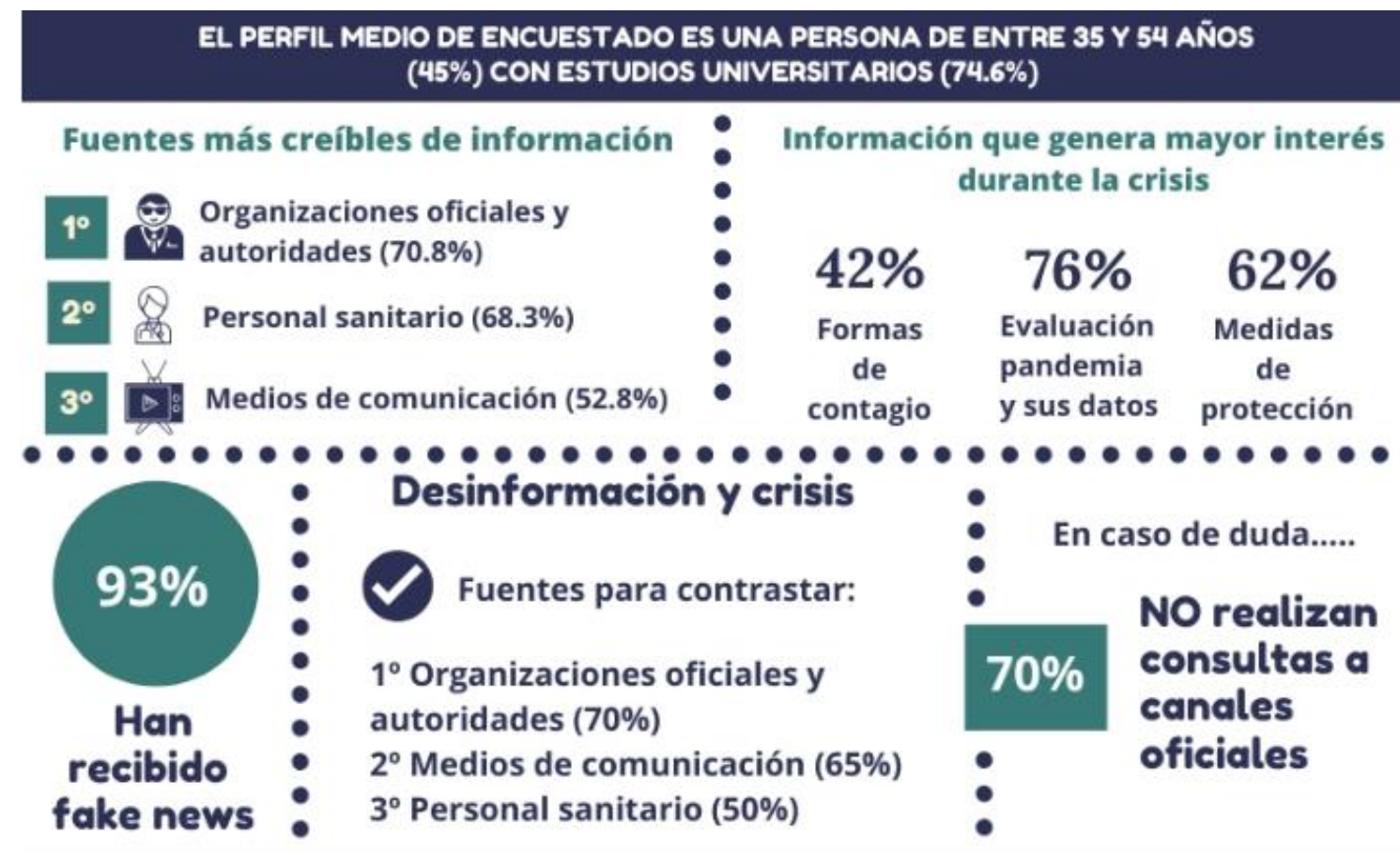

Imagen 1: Infografía resumen del perfil medio de encuestado y sus intereses informativos durante la pandemia.

Fuente: elaboración propia (Canva).

\subsection{Emociones relacionadas con la pandemia y los medios de información}

La dimensión de esta pandemia convierte a esta crisis en un hito histórico a todos los niveles y, hasta el momento, inédito. El Estado de Alarma decretado por el Gobierno y el confinamiento de la población derivado de él, sumado a una situación de incertidumbre en el que la información es un bien de primera necesidad, afecta directamente en las emociones de la población. Así lo demuestra la presente encuesta. La Tristeza (51,5\%) es el sentimiento más mencionado por los encuestados, seguida por la Ansiedad (43,5\%) y el Miedo (42\%). No menos significativo resulta encontrar la Confianza $(23.9 \%)$ como la cuarta emoción más citada. Finalmente, en quinto lugar, estaría la Ira (20.4\%), seguida de la Tranquilidad (11.1\%) y el Orgullo (8\%). La interpretación de estos datos debe contextualizarse en su fase de recogida que se produce durante la primera fase de la crisis, ya que el confinamiento comenzó el 14 de marzo y este estudio se inició 9 días después, el 23 de marzo, extendiéndose hasta el 8 de abril. Igualmente, y como señalan Igartua, Ortega-Mohedano, y ArcilaCalderón. (2020:12) "es probable que la exposición a noticias sobre el número de víctimas del coronavirus estimule emociones negativas (como tristeza o miedo) y esto, a su vez, influye en el recuerdo de la información o en la percepción de la gravedad de la enfermedad".

Dado que el consumo informativo puede estar vinculado con los sentimientos de la población en cada momento, se decidió profundizar en este aspecto a través del cruce de algunas de las variables implicadas, en particular, la que unía estas emociones con el canal de información utilizado. El Miedo se asocia como la emoción predominante con la mayoría de los medios analizados (televisión, 
prensa, WhatsApp, Facebook e Instagram) excepto en las páginas oficiales y la radio, en las que se registra Tranquilidad y en Twitter, red en la que se identifica la Desconfianza.

El Orgullo, la emoción menos mencionada por los encuestados, es también el sentimiento menos asociado a la mitad de los canales (prensa, Twitter, WhatsApp y Facebook). Mientras que los consumidores de televisión sienten en menor grado Desconfianza, los oyentes de radio se desligan de la Tristeza y los usuarios de páginas web oficiales se alejan de la Ira. Resulta llamativo el caso de Instagram, cuyos usuarios tienen la Confianza como la emoción menos vinculada.

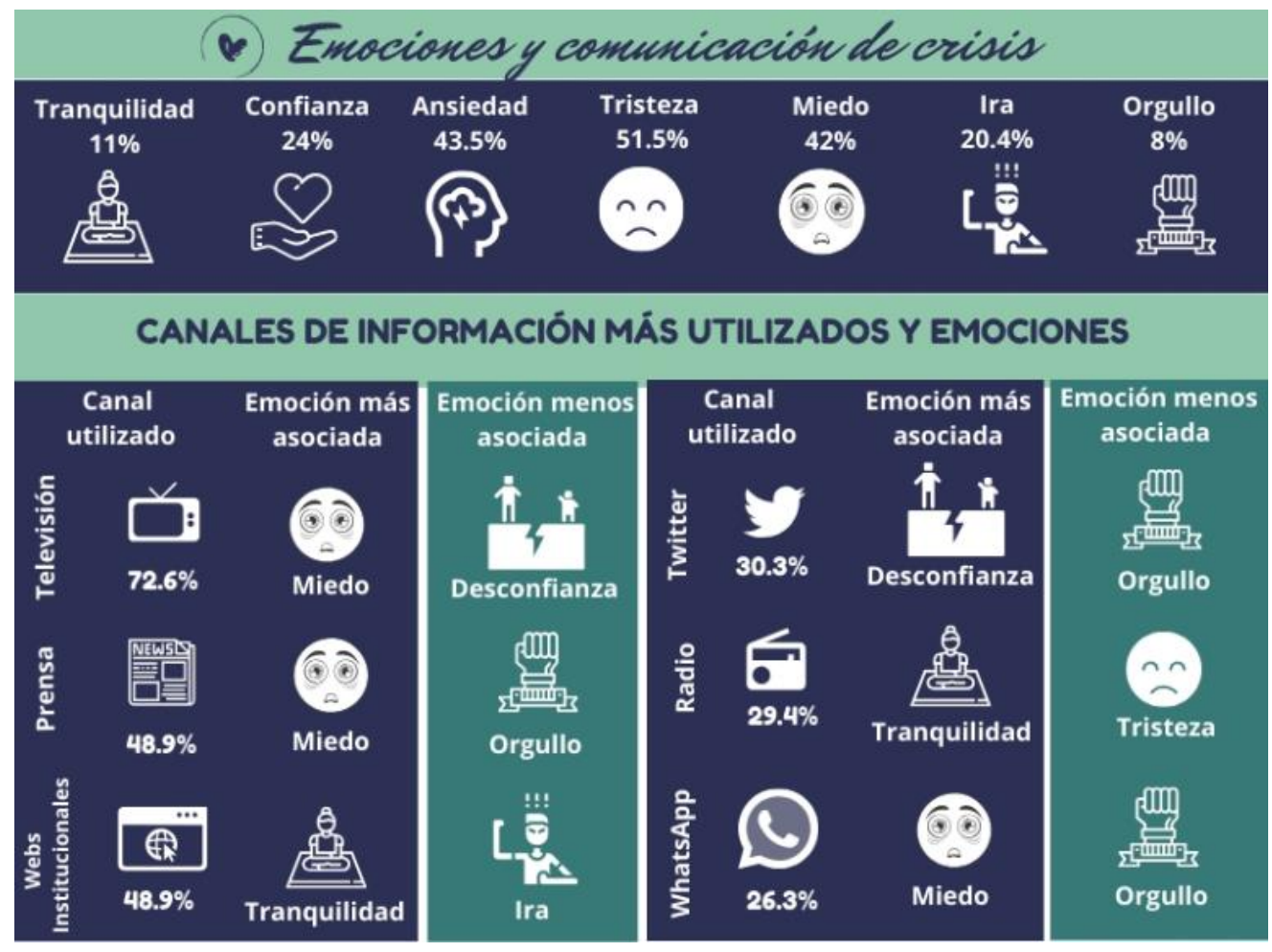

Imagen 2: Infografía resumen de las emociones compartidas por los encuestados durante la crisis.

Fuente: elaboración propia (Canva).

\subsection{Valoración de la comunicación de crisis realizada por el Gobierno}

El $62.31 \%$ de los encuestados aprobaría la gestión de la comunicación de crisis realizada por el Gobierno durante la primera fase de la pandemia, frente al $37.73 \%$ que se muestra disconforme. La mayoría de los encuestados $(52.27 \%)$ puntúa la comunicación del Gobierno durante la crisis entre el 5 y el 8 (en escala de 1 a 10), siendo además ligeramente mayor la valoración en el tramo de entre 7 y 8 puntos $(28,4 \%)$.

Asimismo, se sometieron a evaluación los mensajes recibidos, observándose que no hay gran diferencia entre las tres opciones ofrecidas en la encuesta: información sobre lo que está ocurriendo, soluciones y apoyo emocional. Todas son igualmente apreciadas, y se puntúan entre el 5 y el 7 , siendo la escala de valoración de 1, la menos importante y 7 la más importante. Así, la información sobre soluciones se percibe como el mensaje más importante, ya que un $84 \%$ de los encuestados la evaluaban con un 7. Le siguen los mensajes que ofrecían apoyo emocional que el $77.5 \%$ de los encuestados puntuaba entre 5 y 7 . Resultados muy similares a los mensajes sobre lo que está ocurriendo que el $74.8 \%$ valoró también en el mismo grado. 
Utilizando la escala Likert se ahondó en la percepción sobre algunas características de la información oficial recibida. Los tres aspectos más estimados son la empatía por parte de las autoridades, característica con la que el $57.4 \%$ está muy de acuerdo o de acuerdo, la rapidez (55.3\%) y la claridad de la información (55.2\%). Destaca que el aspecto más apreciado sea puramente emocional, ya que se valora que el Gobierno sea capaz de entender y ponerse en lugar de la ciudadanía por delante de otros items, identificados en comunicación de crisis como esenciales (rapidez y claridad). En contraposición, los ciudadanos no están de acuerdo o están poco de acuerdo con la transparencia ofrecida $(59.7 \%)$ y la veracidad de la información recibida $(59.3 \%)$.

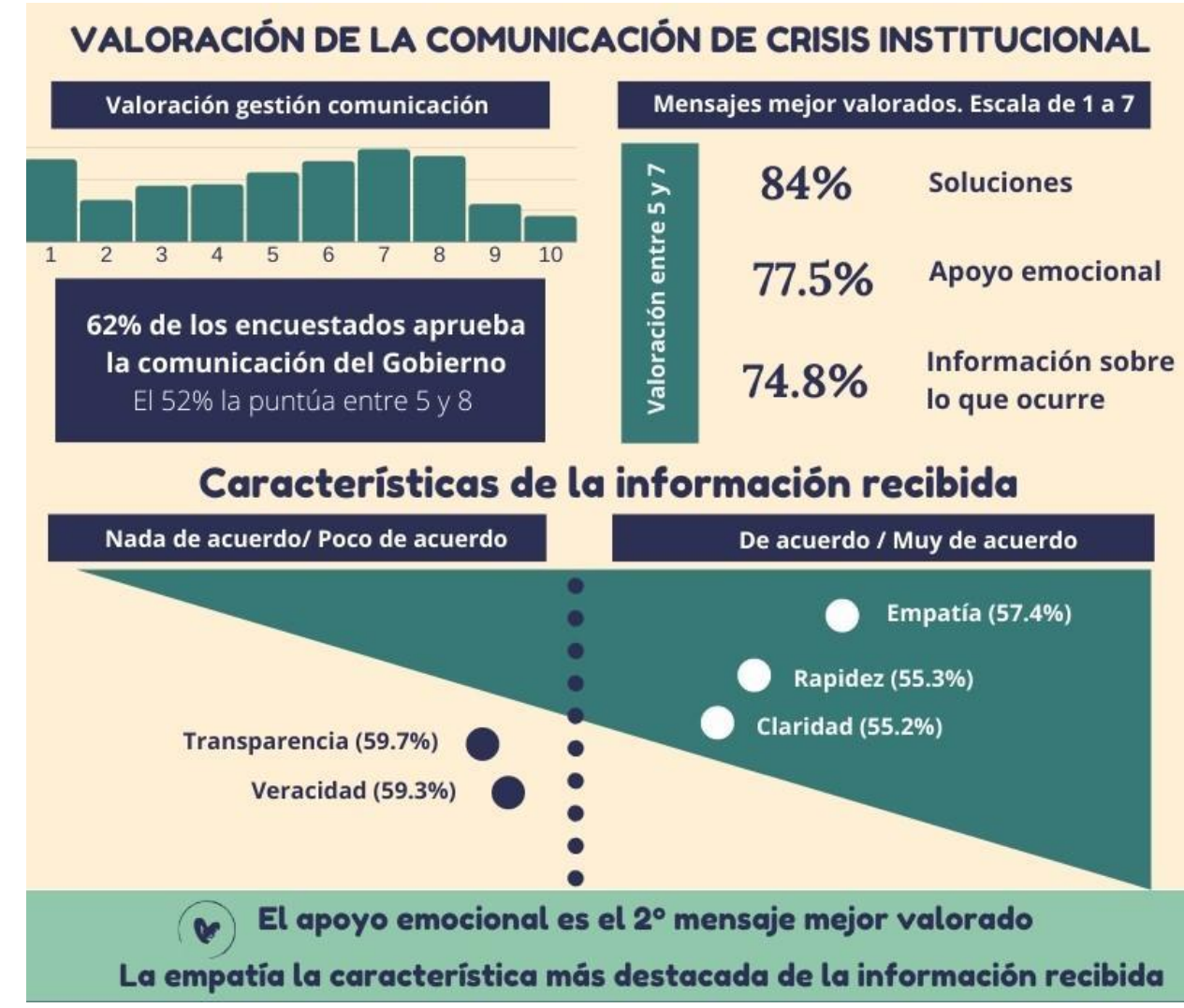

Imagen 3: Infografía resumen de la valoración sobre la comunicación de crisis realizada por el Gobierno durante la primera fase de la pandemia.

Fuente: elaboración propia (Canva)

\section{Discusión y conclusiones}

La dimensión global y las dramáticas consecuencias propiciadas por la irrupción del virus Covid-19 en todo el mundo han hecho necesario poner en marcha mecanismos de comunicación por parte de las autoridades de todos los países. El objetivo del conjunto de Gobiernos ha sido, no sólo de mantener informada a la población en todo momento sino, además, ser capaces de orientar el conjunto de sus emociones para convertirlas en conductas en favor del control de la pandemia y sus devastadores efectos. La crisis del Covid-19, por su gravedad y su urgencia, se han convertido en un paradigma sin precedentes en el que poder analizar el papel de la comunicación en su gestión y en los esfuerzos para tratar de que sus consecuencias sean menos trágicas.

El presente trabajo se centra en el análisis de la influencia de la comunicación oficial de las autoridades (temáticas, contenidos, canales) durante las primeras semanas de la crisis en España en las percepciones de la población, especialmente en su dimensión emocional. Se pretendía encontrar 
una explicación al modo en el que el uso de determinados medios de información y contenidos podría repercutir en los sentimientos de la población y, por ello, en sus comportamientos. De forma complementaria, se ha tratado de cruzar estos datos con la valoración de la opinión pública sobre la información oficial, los temas que despertaban mayor interés y las fuentes más creíbles. También se buscaba saber el conocimiento de los públicos del consumo de fake news y el modo en el que llegaban a contrastar este tipo de informaciones.

Para conocer estos datos se optó por la realización de una encuesta online a partir de un procedimiento no probabilístico durante el periodo inicial del Estado de Alarma decretado en España, desde el 23 de marzo y el 8 de abril de 2020. Se obtuvo una muestra total de 1.823 respuestas entre la población de 18 a 82 años, con residencia en territorio español y acceso a internet.

El trabajo confirma las investigaciones de autores como Coombs (2007), Slovic y Burns (2012), Pang, Jin y Cameron (2009), Van der Meer et al. (2014), Choi y Lin, 2017), Jin, Liu, Anagondahalli y Austin (2014), cuando se refieren al peso decisivo de las emociones en el conjunto de las percepciones de los públicos durante una crisis. En el caso del Covid-19, se confirma este hecho, al comprobarse que son la Tristeza y el Miedo las emociones más presentes en la población durante la primera fase de la pandemia, aunque se observan diferencias en función del canal de comunicación preferente utilizado por la población, circunstancia que podría estar relacionada con la credibilidad de cada canal. Esto explicaría que la radio, canal al que menos se vincula el sentimiento de Desconfianza, ha sido identificada durante varios años como el medio con mayor credibilidad y que, por el contrario, la emoción de Desconfianza asociada a Twitter podría estar relacionada con la desinformación propia del canal, uno de los más frecuentes en la exposición incidental (Serrano Puche et al., 2018; Fletcher \& Nielsen et al., 2018) de los públicos para recibir información.

Las fuentes señaladas como más fiables coinciden con las más utilizadas para contrastar la información, resultando llamativo que se hayan recogido porcentajes prácticamente idénticos en ambas preguntas de la encuesta. Así, las fuentes oficiales se identifican como las creíbles, se valoran en primer lugar como fuentes para contrastar información y se asocia la Tranquilidad a las páginas webs institucionales. Lógicamente se explica esta dependencia a la información institucional dado el carácter sanitario y la magnitud de la crisis, pero resulta significativo que siga existiendo confianza en el discurso oficial y en el trabajo de las instituciones. Más aún, al observar el papel de la desinformación en esta pandemia, que se refleja en que casi todos los encuestados afirman que han recibido fake news. En este punto cabe otra reflexión sobre la responsabilidad individual en torno a la información que se comparte y contrasta durante una crisis, ya que el $70 \%$ de los encuestados reconocía no realizar consultas o contrastar la información. Plantearse las razones de esta actitud, podría abrir nuevas líneas de investigación, tanto si está relacionado con los canales, el carácter de la información o la conformidad informativa de la propia ciudadanía.

El estudio muestra que el $62.31 \%$ de los encuestados aprobaría la gestión de la comunicación de crisis realizada por el Gobierno durante la primera fase de la pandemia, obteniendo una nota media de 7.5 sobre 10. El componente emocional sería, además, una variable importante para esta valoración. En concreto, el apoyo emocional es el segundo mensaje mejor valorado y, la Empatía, la característica más destacada de la información recibida. Se aprecia positivamente la capacidad del Gobierno por "entender" y "ponerse en lugar de la ciudadanía" por delante de otros aspectos, tradicionalmente esenciales en situaciones de crisis, como la rapidez y la claridad en los mensajes emitidos.

Los resultados de este trabajo confirman la importancia del capital emocional de los públicos en la gestión de la información oficial en situaciones de crisis, una variable decisiva para entender el modo 
en el que la población entiende la crisis y se enfrenta a ella. Estas emociones, mayoritariamente negativas, varían en función del canal utilizado y conviven con enormes cantidades de desinformación que los públicos reconocen recibir pero no contrastar. Todos estos hallazgos animan a proseguir con la investigación de las emociones en situaciones de crisis y su anclaje dentro de las políticas de comunicación que deben realizar gobiernos y autoridades en este tipo de contextos. Cabe señalar también que este trabajo supone una primera fase exploratoria que podría complementarse en el futuro con, entre otros, análisis de tipo neurocomunicativo que aporten más información al respecto.

\section{Bibliografía}

Álvarez Fernández, Jesús Timoteo (2009). “Gestión de la comunicación de crisis.” En B. N. O. de E. Iberoamericanos (Ed.), Moreno Castro, Caronia (2009) Comunicar los riesgos. Ciencia y tecnología de la información, Madrid: Biblioteca Nueva Organización de Estados Iberoamericanos. ISBN 9788497429269

Baxter, Leslie A; Babbie, Earl (2004). The basics of communication research. Toronto: Thomson Wadsworth. ISBN: 0534508367

Boyle, Michael; Schmierbach, Mike (2015). Applied Communication Research Methods. New York: Routledge. ISBN: 9781315718644 https://doi.org/10.4324/9781315718644

Choi, Yoonhyeung; Lin, Ying-Hsuan (2009). "Consumer Responses to Mattel Product Recalls Posted on Online Bulletin Boards: Exploring Two Types of Emotion", Journal of Public Relations Research, V.21, N.2, pp.198-207. http://dx.doi.org/10.1080/10627260802557506

Choi, Yoonhyeung; Lin, Ying-Hsuan (2017). "Communicating risk: The effects of message appeal and individual difference on risk message processing". En Annual meeting of the International Communication Association San Francisco, CA. https://doi.org/10.2224/sbp.6327

Coombs, W.-Timothy; Holladay, Sherry J. (1996). "Communication and attributions in a crisis: Anexperimental study of crisis communication". Journal of Public Relations Research, 8, 279-295. https://doi.org/10.1207/s1532754xjprr0804 04

Coombs, W.-Timothy, \& Holladay, Sherry J. (2001). "An extended examination of the crisis situations: A fusion of the relational management and symbolic approaches". Journal of Public Relations Research, 13(4), 321-340. Recuperado de https://www.tandfonline.com/doi/abs/10.1207/S1532754XJPRR1304 03

Coombs, W.-Timothy (2004). "Impact of Past Crises on Current Crisis Communication: Insights From Situational Crisis Communication Theory". Journal of Business Communication, 41(3), 265-289. https://doi.org/10.1177/0021943604265607

Coombs, W.-Timothy; Holladay, Sherry J. (2004). "Reasoned action in crisis communication: An attribution theory-based approach to crisis management". En Dan-P. Millar; Robert -L. Heath (Eds.), Responding to crisis. A Rhetorical approach to crisis communication. Mahwah, NJ: Lawrence Erlbaum, pp. 95-115. ISBN: 0805840605 
Coombs, W.-Timothy (2007). Crisis management and communications, Institute for Public Relations. http://www.instituteforpr.org/topics/crisis-management-and-communications/

Coombs, W.-Timothy; Holladay, Sherry-J. (2008). "Comparing apology to equivalent crisis response strategies: Clarifying apology's role and value in crisis communication". Public Relations Review, 34(3), 252-257, https://doi.org/10.1016/j.pubrev.2008.04.001

Dunlop, Sally; Wakefield, Melanie; Kashima, Yoshi (2008). "Can you feel it? Negative emotion, risk, and narrative in health communication". Media Psychology, 11(1), 52-75. https://doi:10.1080/15213260701853112

Eiroa, Matilde; Barranquero, Alejandro (2017): Métodos de investigación en la comunicación y sus medios. Madrid: Editorial Síntesis. ISBN: 9788490774724

Fletcher, Richard, \& Nielsen, Rasmus Kleis (2018). Are people incidentally exposed to news on social media? A comparative analysis. New Media and Society, 20(7), 2450-2468. https://doi.org/10.1177/1461444817724170

Heath, Robert (2006). "Best Practices in Crisis Communication: Evolution of Practice through Research., Journal of Applied Communication Research, 34(3), 245-248. https://doi:10.1080/00909880600771577

Hilyard, Karen; M., Freimuth; Vicki. S.; Musa, Donald; Kumar, Supriya; Quinn, Sandra-Crouse (2010). "The Vagaries Of Public Support For Government Actions In Case Of A Pandemic". Health Affairs, 29(12), pp.2294-2301. https://doi.org/10.1377/hlthaff.2010.0474

Igartua, J.-J.; Ortega-Mohedano, F. y Arcila-Calderón, C. (2020). Communication use in the times of the coronavirus. A cross-cultural study. El profesional de la información, 29(3). 290-318. https://doi.org/10.3145/epi.2020.may.18

Jin, Yan; Liu, Brooke Fischer; Anagondahalli, Deepa; Austin, Lucinda (2014). "Scale development for measuring publics' emotions in organizational crises". Public Relations Review, 40(3), 509518. https://doi.org/10.1016/j.pubrev.2014.04.007

Karadjov, Christopher; Yungwook, Kim; Karavasilev, Lydmil (2000). "Models of public relations in Bulgaria and job satisfaction among its practitioners". Public Relations Review, 26(2), 209-218. https://doi.org/10.1016/S0363-8111(00)00041-2

Kent, Michael; Taylor, Maureen; Turcilo, Lejla (2006). "Public relations by newly privatized businesses in Bosnia-Herzegovina". Public Relations Review, 32(1), 10-17. https://doi.org/10.1016/j.pubrev.2005.10.001

Kim, Hyo-J; Cameron, Glen-T. (2011). "Emotions matter in crisis: The role of anger and sadness in the publics' response to crisis news framing and corporate crisis response". Communication Research, 38(6), 826-855. https://doi.org/10.1177/0093650210385813

Kleinnijenhuis, Jan; Schultz, Friederike; Oegema, Dirk (2015). "Frame complexity and the financial crisis: A comparison of the United States, the United Kingdom, and Germany in the period 20072012”. Journal of Communication, 65(1), 1-23. https://doi.org/10.1111/jcom.12141 
Lachlan, Kenneth; Spence, Patric; Lin, Xialing; Najarian, Kristy; Del Greco, María (2016). "Social media and crisis management: CERC, search strategies, and Twitter content", Computers in Human Behabior, 54, 647-652, https://doi.org/10.1016/J.CHB.2015.05.027

Lazarus, Richard S. (1991). Emotion and adaption. New York: Oxford University Press, ISBN: 9780195069945

Liu, Broke Fisher.; Fraustino, Julia; Jin, Yan (2015). "How disaster information form, source, type, and prior disaster exposure affect public outcomes: Jumping on the social media bandwagon?". Journal of Applied Communication Research, 43(1), 44-65. https://doi.org/10.1080/00909882.2014.982685

Losada, José Carlos (2018): (NO)CRISIS: comunicación de crisis en un mundo conectado. Barcelona: Ed. UOC. ISBN: 9788491801436

Macias, Wendy; Hilyard, Karen; Freimuth, Vicki (2009). "Blog functions as risk and crisis communication during Hurricane Katrina”. Journal of Computer-Mediated Communication, 15(1), 1-31. https://doi.org/10.1111/j.1083-6101.2009.01490.x

McDonald, Lynette-M; Sparks, Beverley; Glendon, A.-Ian (2010). "Stakeholder reactions to company crisis communication and causes". Public Relations Review, 36(3), 263-271. https://doi.org/10.1016/j.pubrev.2010.04.004

Morrow, John Andrew; Skolits, G. (2014). "The twelve steps of data cleaning: Strategies for dealing with dirty data". Workshop presented at the annual meeting of the American Evaluation Association.

Pang, Augustine; Jin, Yan; Cameron, Glen-T. (2009). "Final stage development of the integrated crisismapping (ICM) model in crisis communication”. En 12th International Public Relations Research Conference, Miami, $\quad$ FL https://ink.library.smu.edu.sg/cgi/viewcontent.cgi?article=7092\&context=lkcsb research

Pang, Augustine; Jin, Yan; Cameron, Glen-T. (2010). "The role of emotions in crisis responses: Inaugural test of the integrated crisis mapping (ICM) model", Corporate Communications An International Journal, 15(4), 428-452 https://10.1108/13563281011085529

Rodríguez-Fernández, L. (2019). Desinformación: retos profesionales para el sector de la comunicación. El profesional de la información, 28(3), 280-306. https://doi.org/10.3145/epi.2019.may.06

Serrano-Puche, J.; Fernández, C. B; Rodríguez-Virgili, J. (2018). Información política y exposición incidental en las redes sociales: un análisis de Argentina, Chile, España y México. Doxa Comunicación, 27, 19-42. $\quad$ Recuperado de https://www.doxacomunicacion.es/es/hemeroteca/articulos?id=273

Schultz, F.; Utz, Sonja, Göritz, Anja (2011): "Is the medium the message? Perceptions of and reactions to crisis communication via twitter, blogs and traditional media". Public Relations Review, 37(1), 20-27. https://doi.org/10.1016/j.pubrev.2010.12.001 
Schwarz, Andreas (2019): "The estate of strategic organizational crisis communication resesarch in the context of global and digital communication". RAEIC, Revista de la Asociación Española de Investigación de la Comunicación, 6(11), 279-310. https://doi.org/10.24137/raeic.6.11.15

Slovic, Paul; Burns, Willian-J. (2012). "Risk perception and behaviors: anticipating and responding to crises". Risk Analysis, 32(4), 579-582. https://doi.org/10.1111/j.1539-6924.2012.01791.x

Stephens, Keri-K.; Malone, Patty (2012). "New Media for Crisis Communication: Opportunities for Technical Translation, Dialogue, and Stakeholder Responses". Coombs, W.-Timothy; Holladay, Sherry-J. (Ed.), The handbook for crisis communication, Chichester (UK): Wiley-Blackwell, pp. 381-395. ISBN: 971405194419

Utz, Sonja; Schultz, Friederike; Glocka, Sandra (2013). "Crisis communication online: How medium, crisis type and emotions affected public reactions in the Fukushima Daiichi nuclear disaster". Public Relations Review, 39(1), 40-46. https://doi.org/10.1016/j.pubrev.2012.09.010

Van der Meer, Toni G.L.A.; Verhoeven, Joost W.M. (2014). "Emotional crisis communication". Public Relations Review, 40(3), 526-536. https://doi.org/10.1016/j.pubrev.2014.03.004

Van Ruler, Betteke; Lange de, Rob (2003). "Barriers to communication management in the executive suite". Public Relations Review, 29(2), 145-158. https://doi.org/10.1016/S0363-8111(03)00017-1

Vázquez-Herrero, J., Vizoso, Á. y López-García, X. (2019). Innovación tecnológica y comunicativa para combatir la desinformación: 135 experiencias para un cambio de rumbo. El profesional de la información, 28(3). 280-301. https://doi.org/10.3145/epi.2019.may.01

Venter, Ben P. (2010). "Views of PRISA members on South African public relations: An exploration". Public Relations Review, 36(3), 281-284. https://doi.org/10.1016/j.pubrev.2010.04.007

Wang, Weirui; Smith, Raquel; Worawongs, Worapron (2010). "Googling the H1N1 Flu: investigating Media Frames in Online News Coverage of the Flu Pandemic". En Annual Meeting of the International Communication Association (Singapur)

Wendling, Cécile; Radisch, Jack; Jacobzone, Stephane (2013). "The Use of Social Media in Risk and Crisis Communication", OECD Working Papers on Public Governance, 25, OECD Publishing. http://dx.doi.org/10.1787/5k3v01fskp9s-en

Wesseling, Yoka-M.; Kerkhof, Peter; Van Dijk, Wilco-W. (2006). "Communicatie van emoties bij organisatiecrisis: Differentiële effecten van spijt en schaamte". Jaarboek Sociale Psychologie, ASPO pers, pp. 539-550.

Wu, Ming-Yi; Taylor, Maureen (2003). "Public relations in Taiwan: Roles, professionalism, and relationship to marketing". Public Relations Review, 29(4), 473-483. https://doi.org/10.1016/j.pubrev.2003.08.008 


\section{AUTORES}

\section{José Carlos Losada}

José Carlos Losada es Profesor Titular de Comunicación Institucional y Corporativa de la Universidad de Murcia (España. Ha publicado tres libros, entre los que destaca Comunicación en la Gestión de Crisis: lecciones prácticas, Editorial UOC (2018) o (NO) CRISIS, Editorial UOC (2010), además de la coordinación de Gestión de la Comunicación en las Organizaciones, Ed.Planeta (2004). Igualmente, es autor de 20 textos en libros colectivos para editoriales de varios países: Springer, Fragua, Executive Business School, Universidad Complutense de Madrid, etc. Ha publicado 30 textos en Journals indexados de todo el mundo, como El Profesional de la Información, Central Journal of Communication, Palabra Clave, Journal of Public Health Research, Razón y Palabra, Comunicación y Sociedad...Ha participado en diferentes de proyectos de Investigación nacionales (Ministerio de Educación y Ciencia) e Internacionales (Comisión Europea), además de contratos de investigación para empresas. En el ámbito profesional ha trabajado como consultor de comunicación e imagen, ha asesorado para más de 20 organizaciones políticas, empresariales, marcas y gobiernos durante los últimos 23 años.

jclosada@um.es

Orcid ID: https://orcid.org/0000-0002-3966-837X

Google Scholar: https://scholar.google.com/citations?user=sHUjujEAAAAJ\&hl=en

\section{Leticia Rodríguez Fernández}

Leticia Rodríguez Fernández es investigadora y docente en el área de comunicación organizacional en la Universidad Antonio Nebrija, donde dirige el Grado en Comunicación Corporativa, Protocolo y Organización de Eventos. Imparte, entre otras asignaturas, «Pensamiento crítico ante las fake news», «Comunicación corporativa» y «Comunicación institucional». Doctora en Ciencias de la Información (UCM, 2012), Máster Universitario en Protocolo (UNED, 2019), Máster en Comunicación de Instituciones Públicas y Políticas (UCM, 2008) y Licenciada en Publicidad y Relaciones Públicas (UVA, 2007). Antes de dedicarse a la docencia, trabajó en varios departamentos de comunicación y agencias de relaciones públicas. Sus líneas de investigación se centran en la comunicación organizacional, con especialización en el área digital y el estudio de la propaganda y la desinformación.

lrodrigu@nebrija.es

Orcid ID: https://orcid.org/0000-0002-7472-5472

Google Scholar: https://scholar.google.es/citations?user=8slP_GUAAAAJ\&hl=es

\section{Francisco Javier Paniagua Rojano}

Francisco Javier Paniagua Rojano es Profesor Titular en el Departamento de Periodismo en la Universidad de Málaga, donde imparte Comunicación Institucional y Corporativa. Entre sus líneas de investigación destacan comunicación organizacional, social media y credibilidad de los medios. Es coordinador de la sección Comunicación estratégica y organizacional en la Asociación Española de Investigación en Comunicación (AEIC). Ha sido Director de Comunicación de la Federación Andaluza de Municipios y Provincias (FAMP) y la Universidad Internacional de Andalucía (UNIA). fjpaniagua@uma.es

Orcid ID: http://orcid.org/0000-0001-7376-4536

Google Scholar: https://scholar.google.es/citations?user=9z8ZuMMAAAAJ 